\title{
Small-Scale Anti-Parallel Field-Aligned Currents and Plasma Density Dips: Signatures of the "Alfvénic Shocks"
}

\author{
E. V. MISHIN ${ }^{1}$, M. FöRSTER ${ }^{2}$, and D. POPESCU ${ }^{3}$ \\ ${ }^{1}$ Max-Planck-Institut für Aeronomie, Postfach 20, D-37189 Katlenburg-Lindau, Germany \\ ${ }^{2}$ Max-Planck-Institut für extraterrestrische Physik, Außenstelle Berlin, Rudower Chaussee 5, D-12489 Berlin, Germany \\ ${ }^{3}$ Institute of Gravitation and Space Sciences, P.O. Box MG-6, R-76900 Bukarest, Romania
}

(Received December 8, 1994; Revised December 1, 1995; Accepted November 14, 1997)

\begin{abstract}
"Electrostatic Shocks" and field-aligned currents are associated with discrete auroral arcs and lowaltitude particle acceleration. We show that accounting for the two new elements with respect to the previous "static" and "Alfvén wave" models, namely the transverse wave-guide for shear Alfvén waves and plasma density dip in the center of the structure, is necessary in order to explain the basic features of this phenomenon. The characteristics dimensions of the structure correspond to the "stirring scales" of the auroral turbulence.
\end{abstract}

\section{Introduction}

Small-scale, $l_{\perp}<10 \mathrm{~km}$, auroral arcs are known to follow strong oppositely-peak-shaped, N-S electric fields ("electrostatic shocks" (Mozer et al., 1977)) and U-shaped, E-W magnetic perturbations (pairs of field-aligned currents $j_{z}$ ), accompanied by intense shear plasma flows (Burke et al., 1983; Heelis et al., 1984; Volokitin et al., 1984; Weimer et al., 1985; Chmyrev et al., 1985; Dubinin et al., 1988) and upward $\mathrm{O}^{+}$fluxes (Heelis et al., 1984). Henceforth, these $\boldsymbol{E}-\boldsymbol{B}$ or $\boldsymbol{E} \propto j_{z}$ structures are referred to as "Alfvénic Shocks" (ASs), emphasizing the decisive role of (shear) Alfvén waves and collisionless dissipation in their formation (Mishin et al., 1993; Mishin and Förster, 1995). Clearly, the ASs are associated with the low-altitude acceleration.

The "static model (Weimer et al., 1985) interprets such a shock as a pair of oppositely-directed, turbulent field-aligned current sheets $(\partial / \partial x>>\partial / \partial z, \partial / \partial y=0)$, closed by the Pederson current in the $E$ region. It does not fit the data, when approaching the small scales characteristics of the ASs (cf. e.g., Ishii et al., 1992).

In the "Alfvén wave" model (Haerendel, 1983; Lysak and Dum, 1983), field-aligned currents $j_{z}$ (FACs), are closed by the polarization current in shear, $k_{x}>>k_{z, y}$, Alfvén waves (SAWs). Their existence in the auroral ionosphere has been securely established (e.g., Gurnett et al., 1984; Dubinin et al., 1988; Boehm et al., 1990; Ishii et al., 1992). However, the transverse group velocity of short-scale SAWs, of interest, is rather high, constradicting the AS's stationarity. Further, the electric and magnetic field relation in a plane up/downward $(\uparrow / \downarrow)$ propagating SAW,

$$
E_{x}^{\uparrow \downarrow}= \pm\left(V_{\mathrm{A}} / c\right) B_{y} \quad\left(V_{\mathrm{A}} \text { is the Alfvén velocity }\right)
$$

yields $E_{x} \neq 0$ when $j_{z} \propto \partial B_{y} / \partial x=0$. If incident $(\downarrow)$ and reflected $(\uparrow)$ waves interfere, $E_{x}=E_{x} \uparrow+E_{x}{ }^{\downarrow} \simeq 0$, but $j_{z}=j_{z}{ }^{\downarrow}+j_{z}{ }^{\uparrow} \simeq 2 j_{z}{ }^{\downarrow} \neq 0$. Therefore, matching $j_{z}$ and $E_{x}$ in the AS-topology, i.e., $E_{x} \propto j_{z}$, is impossible unless (1) is changed. We show, in accord with (Mishin and Förster, 1995), that the ASs are accompanied by strong plasma density reductions and the coupling of shear Alfvén waves with density perturbations, with a proper account of the anomalous resistance, allows to rule out these "bottlenecks". 


\section{On Plasma Density Reductions}

Strong density reductions at heights $\sim(1-2) \mathrm{R}_{\mathrm{E}}$ accompany "electrostatic shocks" (ESs) (Mozer et al., 1977) and narrow upward ion beams (Persoon et al., 1988; Hultqvist, 1991). The most prominent "cavities" map to scales $\gtrsim 100 \mathrm{~km}$ at the ionosphere's height, while the ESs are related to weaker pronounced 'holes'. One can also see that the cavities (Mozer et al., 1977; Persoon et al., 1988; Hultqvist, 1991) consist of several sub-structures.

The low-altitude $n$-depletions, connected with intense SAWs and upward large-scale FACs, have

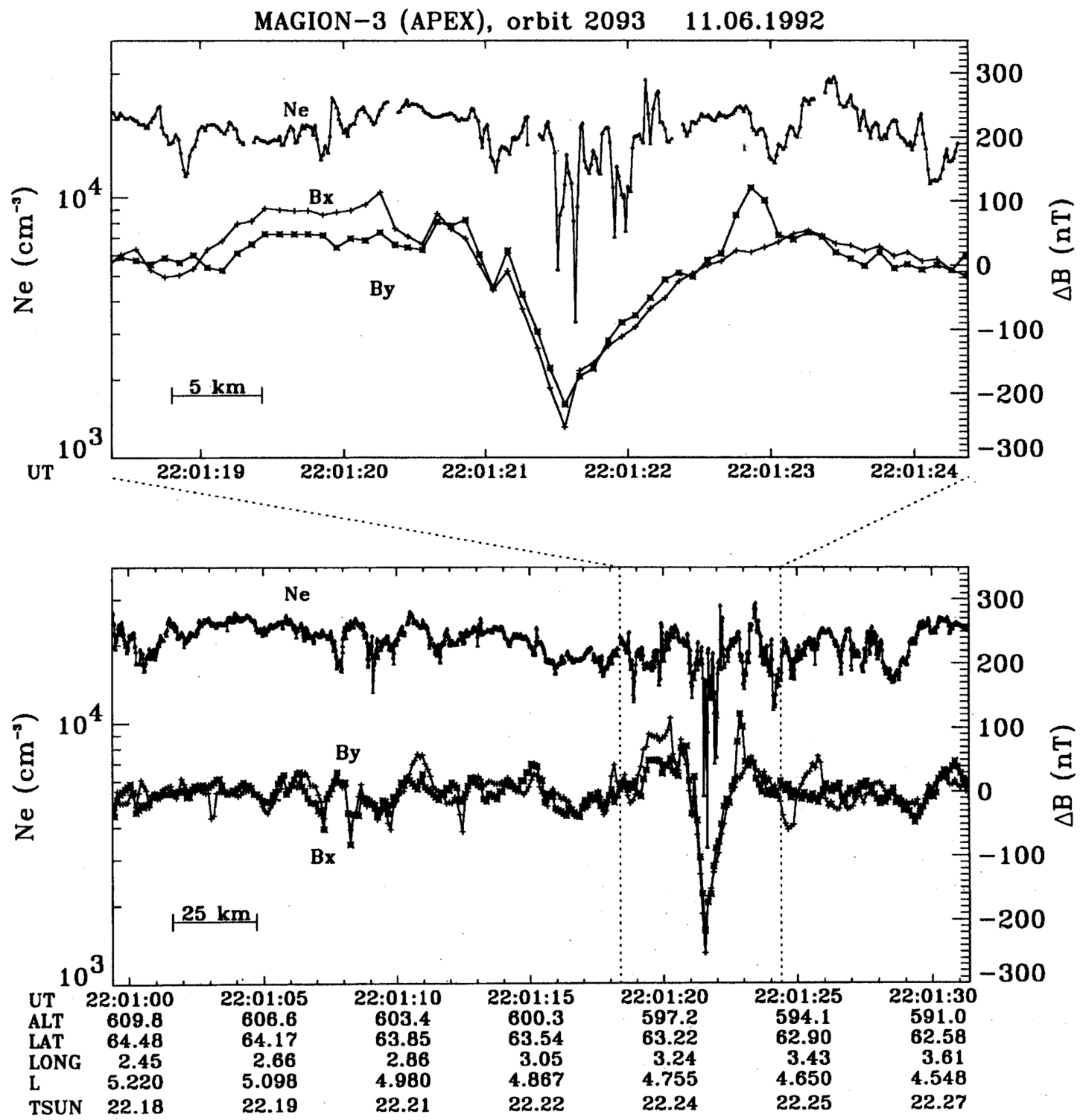

Fig. 1. Examples of plasma density reductions together with magnetic field perturbations at low altitudes: Magion-3 (Apexmission), orbit 2093 on June 11, 1992. 
been reported (Boehm et al., 1990) and (Abe et al., 1993). The width of the deepest, $\mathrm{A}=\bar{n} / n_{\mathrm{dip}}-1 \sim 10$, 'dip' is of the order of $\lambda_{1} \leq 500 \mathrm{~m}$. In its neighbourhood there are several moderately depleted 'holes', $\lambda_{2}$ $\leq(5-10) \lambda_{1}$. Two examples of similar but less depleted dips, associated with ion energization events, have been discussed by Vago et al. (1992).

Figure 1 and 2 show examples of density reductions detected by the Langmuir probe onboard Magion 3 (Apex mission) and Magion 2 (Aktivny) spacecrafts during intense small-scale magnetic perturbations.

Magion 2 and 3 as subsatellites of the Aktivny and Apex missions operated at a near polar orbit (inclination about 82.5 degrees) in the upper ionosphere. Their apogee and perigee were about 2500/500 $\mathrm{km}$ and 3000/400 km, respectively. The time interval of active operations of Magion 2 in orbit was from

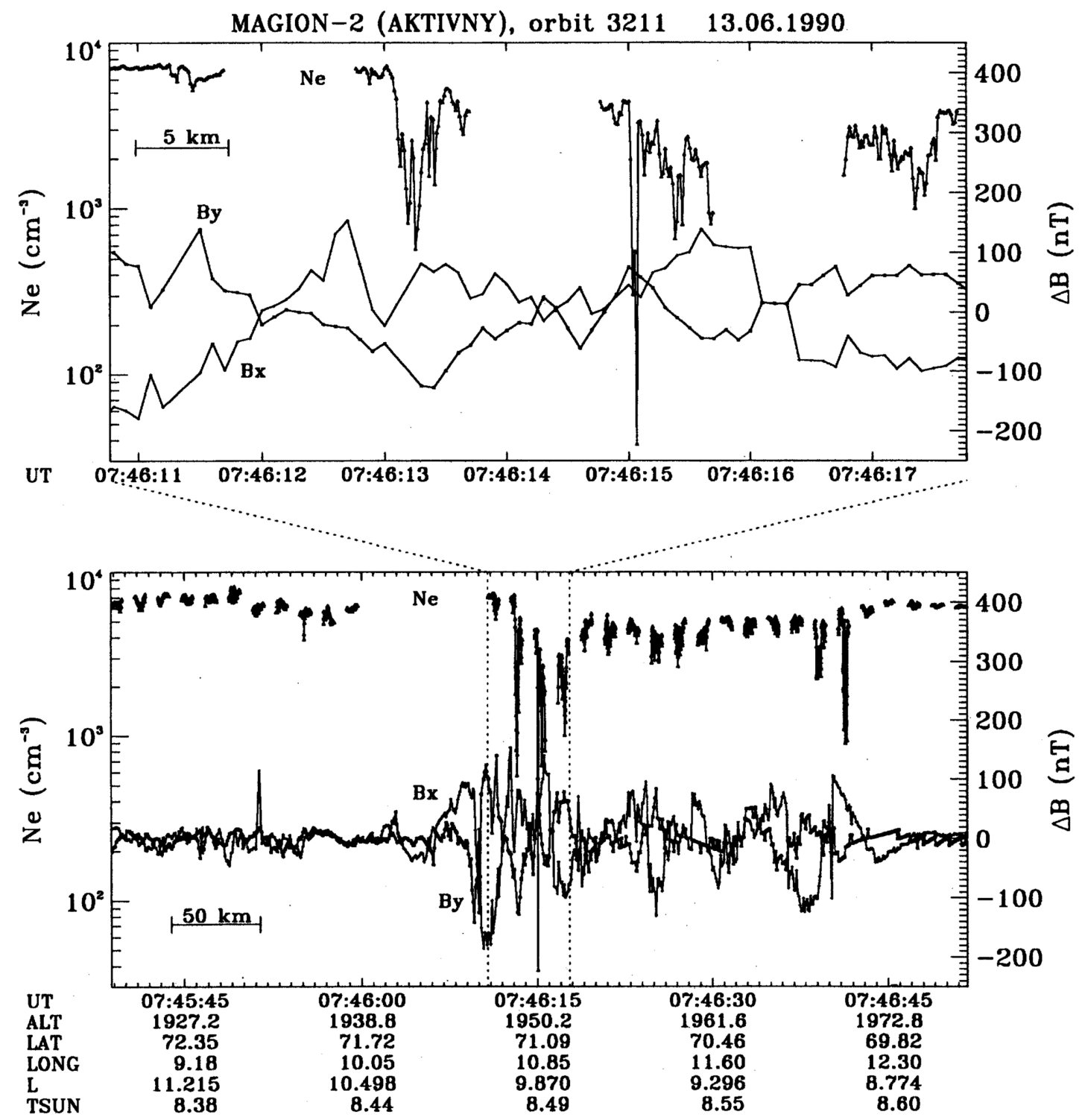

Fig. 2. Plasma density depletions observed by Magion-2 (Aktivny) on June 13, 1990 at an altitude of $1950 \mathrm{~km}$ together with magnetic field perturbations. 
January 1990 till October 1990 and for Magion 3 from December 1991 till August 1992. A description of the equipment and the scientific tasks of Magion 2 and 3 subsatellites can be found in the paper of Triska et al. (1990).

Electron density depletions were observed by the cylindrical Langmuir probe ZLA in constant voltage regime. Magion 2 and 3 satellites are magnetically oriented and so the main axis of the Langmuir probe is approximately parallel to the geomagnetic field lines. A positive voltage of $4 k_{\mathrm{B}} T_{\mathrm{e}}$ (about $2 \mathrm{eV}$ ) is applied to the cylindrical Langmuir probe and the fluctuating current measured is interpreted as electron density fluctuations while any other influences (like plasma potential variations or electron temperature fluctuations) are neglected. This is justified for a dense thermal plasma like that of the upper ionosphere (cf. Boström, 1992). Digital data of electron density variations are taken with a time resolution of $64 \mathrm{~Hz}$. A complete description of the Langmuir probe ZLA working regimes was given by Rustenbach (1986).

The electron density dips are accompanied by intense field-aligned currents as seen by the magnetometer SGR. This Romanian probe is a three-axial fluxgate magnetometer which allows a time resolution of $20 \mathrm{~Hz}$. Additionally, for one component $\left(\Delta B_{x}\right)$ a variometer data output is installed. More details can be found in the paper of Ciobanu et al. (1993).

Figure 1 shows an example of density reductions detected by the Langmuir probe onboard Magion 3 spacecraft (orbit 2093). The density depletion in this case is about 80 per cent within a horizontal distance of about $500 \mathrm{~m}$. During the same time intense small-scale magnetic perturbations are observed in the components perpendicular to the main geomagnetic field $\left(B_{x}\right.$ and $\left.B_{y}\right)$. The angle of both $x$ - and $y$-components with regard to the orbital trajectory is about 45 degrees, so that the magnetic deviations are nearly equal. The FACs in this event reach about $100 \mu \mathrm{A} \mathrm{m} \mathrm{m}^{-2}$.

Similar events have been also seen by Magion 2. Figure 2 shows an example of orbit 3211 from June 13,1990 . This event occurred during strongly disturbed geomagnetic conditions and was observed at an altitude of about $1950 \mathrm{~km}$ in the morning sector. The electron density dip in the most extreme case reaches two orders of magnitude and the disturbed region confines to horizontal distances of a few hundred meters to 1-2 kilometers. The FAC density in these cases is about $\sim 50-75 \mu \mathrm{A} \mathrm{m}^{-2}$.

\section{On Alfvén Wave Model}

In the low $-\beta(<<m / M)$ plasma, SAWs may be trapped inside density depletions $\perp \vec{B}_{0}$, serving as a transverse-Alfvén-wave-guide (TAWG) (Gul'elmi and Polyakov, 1983). Thus, pairs of small-scale antiparallel FACs are to be the eigenmodes of the TAWG, i.e., a density cavity at heights $\leq 2 R_{E}$ (Mishin et al., 1993; Mishin and Förster, 1995), suggesting a local source of SAWs (cf. Heppner et al., 1993). The 'TAWGrelation' $\lambda_{\perp} \sim \sqrt{L_{\perp} d}$ links quite resonably the scales of the "cavity" $L_{\perp}$ and "holes" $\lambda_{\perp}$, where $d=c / \omega_{\mathrm{p}}$ $\simeq 5 n^{-1 / 2} \mathrm{~km}$ is the plasma skin depth.

Further, (1) follows from the current continuity, when $\partial E_{x} / \partial t \rightarrow i \omega E_{x}$. More generally, one should use $d / d t=\partial / \partial t+u_{x} \partial / \partial x$, where $u_{x}$ is the AS velocity relative to the background plasma (Haerendel et al., 1993). Assuming the spatial derivative dominating, yields

$$
\frac{\partial}{\partial x} E_{x}=\frac{4 \pi V_{\mathrm{A}}^{2}}{c^{2} u_{x}} j_{x}=\frac{V_{\mathrm{A}}^{2}}{c u_{x}} \frac{\partial}{\partial z} B_{y} \propto \frac{B_{y}}{n u_{x}} .
$$

That is, in a steady state $(\omega \sim \partial / \partial t \rightarrow 0)$ the relation (1) breaks down. If at $x \rightarrow 0, n \rightarrow 0$ or $n(x)^{-1} \propto \delta(x)$, clearly, $E_{x} \propto \operatorname{sgn}(x)$, i.e., the dip in the center permits matching at $x \rightarrow 0$ the oppositely-directed (ES) electric fields. In turn, from the ion continuity equation one gets $n_{\mathrm{i}}(x)=\operatorname{const}\left(\partial E_{x} / \partial x\right)^{-1}$. Therefore, the plasma density has actually to be depleted in the region of large gradients of $E_{x}$, i.e., a the border between the AS-electric fields. This implies the increase of the plasma transport perpendicular to the magnetic field as a result of instabilities due to the either shear flow $u_{y}(x)=c E_{x}(x) / B_{0}$, if $\Omega_{\mathrm{s}} \gtrsim \Omega_{\mathrm{i}}$ (e.g., G. Ganguli et al., 1994) or/and transverse current (e.g., Volokitin et al., 1984) when $\left|u_{x}\right| \geqslant v_{T_{i}} \Omega_{i} / \Omega_{\mathrm{s}}$. Here $\Omega_{\mathrm{s}}=\partial u_{y} / \partial x$ is the 
shear frequency and $\Omega_{\mathrm{i}}$ is the ion gyrofrequency.

On the other hand, the steady state reaches (Haerendel, 1983) when $\tau_{\text {dif }} \sim l_{\perp}{ }^{2} / d^{2} v_{\text {ef }}<<l_{\perp} /\left|u_{x}\right| ; l_{\|} / V_{\mathrm{A}}$, implying that the electromagnetic AS-structure can be reduced to the quasi-electrostatic one (Haerendel, 1983; Mishin and Förster, 1995)

$$
-\Phi(x)=\int d x^{\prime} E_{x} \approx \int d z^{\prime} \frac{j_{z}\left(x, z^{\prime}\right)}{\sigma_{\|}\left(x, z^{\prime}\right)} \Rightarrow B_{y} \approx \frac{\tilde{a}}{\mu_{0}} \int E_{x} d x^{\prime} d x^{\prime \prime} \quad \text { if } \frac{\sigma_{\|}}{l_{\|}}=\operatorname{const}(x) .
$$

Here $\sigma_{\|} \propto n / v_{\mathrm{ef}}$ is the anomalous conductivity; $-j_{z} / \Phi=\tilde{a} \approx \sigma_{\|} / l_{\|} ; l_{\|} \sim$ a few $1000 \mathrm{~km}$ is the longitudinal extent of the acceleration region.

The relation (3) fits the data at heights $\sim \mathrm{R}_{\mathrm{E}}$ for $\tilde{a} \sim 3 \cdot 10^{-8} \mathrm{mho} / \mathrm{m}^{2}$ (Weimer et al., 1985), corresponding to $v_{\mathrm{ef}} \sim n\left(1000 \mathrm{~km} / l_{\|}\right) \gg v_{\mathrm{ei}} \sim 10^{-5} n$. Clearly, the anomalous resistivity in the region $j_{z} \rightarrow 0$ can be caused by the above "transverse" instabilities, resulting in excitation of lower-hybrid waves (LH), energizing some amount of particles (e.g., Bingham et al., 1987; Vago et al., 1992). Apart from that, ions gain the transverse energy, when moving in the inhomogeneous electric field. Thus, the energy of the transverse current/flow is transformed into the accelerated particles inside the dip.

Furthermore, the density decrease by a factor of $\gtrsim 10$ in the dip should lead to Buneman instability of the upflowing Birkeland current $j_{z}^{(B)}$, when $j_{z}{ }^{(B)} / n_{\text {dip }} \geqslant j_{z}{ }^{(\max )} / \bar{n}>u_{\|}^{\text {thr }} \rightarrow v_{T_{\mathrm{e}}}$. It yields asymmetry of $E_{z}(x)$ and $E_{x}(x)$, so that $\int_{-l_{1}}^{+l_{1}} E_{z, x} d x \neq 0$. Clearly, electrons can be accelerated by the longitudinal electric field either up or down, depending of the $\operatorname{sgn}\left(j_{z}{ }^{B}\right)$.

It is noteworthy that the (large-scale) Birkeland current should form an initial depletion (e.g., Block and Fälthammar, 1968), i.e., the "seed" of a cavity, emphasizing also the $\| \vec{B}_{0}$-Alfvén resonator, needed for the excitation of small-scale SAWs due to the instability of the magnetosphere-ionosphere coupling (Trakhtengertz and Feldstein, 1991). After the onset of the energization, the depletion should deepen further (e.g., Haerendel, 1993; Singh, 1994; S. Ganguli et al., 1994).

Concluding, note that recent studies of the irregular auroral electric field (e.g., Earle and Kelley, 1993) have shown the existence of the stirring range, 300-500 $\mathrm{m} \leqslant \lambda_{\perp} \leqslant 2-3 \mathrm{~km}$, in the regions of intense small-scale shear flows, $\Omega_{\mathrm{s}} \gtrless 10 \mathrm{~s}^{-1}$ at heights $\sim 500 \mathrm{~km}$. Earle and Kelley (1993) have emphasized that this spectral range '... matches the frequency range $~ 0.2$ to $\sim 1 \mathrm{~Hz}$ typical for the Alfvén waves which could be the source of plasma stirring as spatial shears'. On the basis of the foregoing consideration, we suggest that the stirring-range is the intrinsic range for the ASs.

This paper is partly supported by the Deutsche Agentur für Raumfahrtangelegenheiten (DARA) GmbH under grant 50 QL 92060.

\section{REFERENCES}

Abe, T., K.-I. Oyama, S. Watanabe, and H. Fukunishi, Characteristic features of electron temperature and density variations in field-aligned current regions, J. Geophys. Res., 98, 11,257-11,266, 1993.

Bingham, R., D. Bryant, and D. Hall, Auroral electron acceleration by lower-hybrid waves, Ann. Geophys., 6, 159-168, 1987.

Block, L. and C.-G. Fälthammar, Effects of field-aligned currents on the structure of the ionosphere, J. Geophys. Res., 73, 4807$4812,1968$.

Boehm, M., C. Carlson, J. McFadden, J. Clemmons, and F. Mozer, High-resolution sounding rocket observations of largeamplitude Alfvén waves, J. Geophys. Res., 95, 12,157-12,171, 1990.

Boström, R., Observations of weak double layers on auroral field lines, IEEE Transactions on Plasma Science, 20, No. 6, 756$763,1992$.

Burke, W., M. Silevitch, and D. Hardy, Observations of small-scale auroral vortices by the S3-2 satellite, J. Geophys. Res., 88, 3127-3137, 1983.

Chmyrev, V., V. Oraevsky, S. Bilichenko, N. Isaev, G. Stanev, D. Teodosiev, and S. Shkolnikova, The fine structure of intensive small-scale electric and magnetic fields in the high-latitude ionosphere as observed by INTERKOSMOS-BULGARIA 1300 
satellite, Planet. Space Sci., 33, 1383-1388, 1985.

Ciobanu, M. I., H. Comisel, E. Georgescu, D. Popescu, A. Moldovanu, M. Macoviciuc, E. Moldovanu, and E. Diaconu, Auroral geomagnetic events catalogue (Magion-Intercosmos 24), Institute of Atomic Physics and Institute of Gravitation and Space Sciences, Report, Bukarest, 1993.

Dubinin, E. M., A. S. Volokitin, P. L. Izrailevich, and N. S. Nikolaeva, Auroral electromagnetic disturbances at altitudes of 900 km: Alfvén wave turbulence, Planet. Space Sci., 36, 949-962, 1988.

Earle, G. D. and M. C. Kelley, Spectral evidence for stirring scales and two-dimensional turbulence in the auroral ionosphere, J. Geophys. Res., 98, 11,543-11,548, 1993.

Ganguli, G., M. Keskinen, H. Romero, R. Heelis, T. Moore, and C. Pollock, Coupling of microprocesses and macroprocesses due to velocity shear: An application to the low-altitude ionosphere, J. Geophys. Res., 99, 8873-8889, 1994.

Ganguli, S., H. Mitchell, and P. Palmadesso, Correlated low-frequency electric and magnetic noise along the auroral field lines, J. Geophys. Res., 99, 5761-5770, 1994.

Gul'elmi, A. and A. Polyakov, On the discontinuous spectrum of Alfvén oscillations, Geomagn. Aeron. (Engl. transl.), 23, 281$282,1983$.

Gurnett, D., R. Huff, J. Menietti, J. Burch, J. Winningham, and S. Shawhan, Correlated low-frequency electric and magnetic noise along the auroral field lines, J. Geophys. Res., 89, 8971-8985, 1984.

Haerendel, G., An Alfvén wave model of auroral arcs, in High-Latitude Space Plasma Physics, edited by B. Hultqvist and T. Hagfors, pp. 515-535, Plenum Press, New York, 1983.

Haerendel, G., Acceleration from field-aligned potential drops, MPE Preprint 250, 35 pp., March, 1993.

Haerendel, G., S. Buchert, C. La Hoz, B. Raaf, and E. Rieger, On the proper motion of auroral arcs, J. Geophys. Res., 98, 6087$6099,1993$.

Heelis, R., J. Winningham, M. Sugiura, and N. Maynard, Particle acceleration parallel and perpendicular to the magnetic field observed by DE 2, J. Geophys. Res., 89, 3893-3902, 1984.

Heppner, J. P., M. C. Liebrecht, N. C. Maynard, and R. F. Pfaff, High-latitude distributions of plasma waves and spatial irregularities from DE-2 alternating current electric field observations, J. Geophys. Res., 98, 1629-1652, 1993.

Hultqvist, B., Extraction of ionospheric plasma by magnetospheric processes, J. Atmos. Terr. Phys., 53, 3-15, 1991.

Ishii, M., M. Sugiura, T. Iyemori, and J. Slavin, Correlation between magnetic and electric field perturbations in the field-aligned current regions deduced from DE 2 observations, J. Geophys. Res., 97, 13,877-13,887, 1992.

Lysak, R. L. and C. T. Dum, Dynamics of magnetosphere-ionosphere coupling including turbulent transport, J. Geophys. Res., 88, 365-380, 1983.

Mishin, E. V. and M. Förster, 'Alfvénic shocks' and low-altitude auroral acceleration, Geophys. Res. Lett., 22, $1745-1748,1995$.

Mishin, E. V., J. Büchner, and M. Förster, Anti-parallel field-aligned currents as indicators of eigenmodes of transverse Alfvén resonances, AGU 1993 Fall Meeting, EOS Suppl., Oct. 26, 541, 1993.

Mozer, T. S., C. Carlson, M. Hudson, R. Torbert, B. Parady, and J. Yatteau, Observations of paired electrostatic shocks in the polar ionosphere, Phys. Rev. Lett., 38, 292-295, 1977.

Persoon, A., D. Gurnett, W. Peterson, J. Waite, Jr., J. Burch, and J. Green, Electron density depletions in the nightside auroral zone, J. Geophys. Res., 93, 1871-1895, 1988.

Rustenbach, J., Gerätekonzeption und Systemlösung eines modular aufgebauten adaptiven Langmuirsondenmeßsystems als Bestandteil des plasmadiagnostischen Gerätekomplexes der Satellitenmission 'Aktivny', Thesis, Akademie der Wissenchaften, Berlin, 1986.

Singh, N., Ponderomotive versus mirror force in creation of the filamentary cavities in auroral plasma, Geophys. Res. Lett., 21, 257-260, 1994.

Trakhtengertz, V. and A. Feldstein, Turbulent Alfvén boundary layer in the polar ionosphere, I. Excitation conditions and energetics, J. Geophys. Res., 96, 19,363-19,374, 1991.

Triska, P., F. Jiricek, V. Velicky, and J. Vojta, A subsatellite for mother-daughter active experiments, Adv. Space Res., 10, 165$169,1990$.

Vago, J., P. Kintner, S. Chesney, R. Arnoldy, K. Lynch, T. Moore, and C. Pollock, Transverse ion acceleration by localized lower hybrid waves in the topside auroral ionosphere, J. Geophys. Res., 97, 16,935-16,957, 1992.

Volokitin, A., V. Krasnoselskikh, E. Mishin, L. Tyurmina, V. Sharova, and S. Shkol'nikova, Small-scale structure of intense longitudinal currents in high latitudes, Kosm. Issled. (Space Researches; Engl. transl.), 22, 613-619, 1984.

Weimer, D., C. Goertz, D. Gurnett, N. Maynard, and J. Burch, Auroral zone electric fields from DE 1 and 2 at magnetic conjunctions, J. Geophys. Res., 90, 7479-7494, 1985. 